INPLASY

PROTOCOL

To cite: Liang et al. Chinese

herbal medicine combined with acupuncture for women with polycystic ovarian syndrome. Inplasy protocol 202180048. doi:

10.37766/inplasy2021.8.0048

Received: 12 August 2021

Published: 12 August 2021

Corresponding author:

Hongxia Ma

doctorhongxia@126.com

Author Affiliation:

The First Affiliated Hospital Of

Guangzhou Medical University.

Support: National Natural

Science Found.

Review Stage at time of this submission: Formal screening of search results against

eligibility criteria.

Conflicts of interest:

None declared.

\section{Chinese herbal medicine combined with acupuncture for women with polycystic ovarian syndrome}

Review question / Objective: To assess the effectiveness and safety of CHM combined with acupuncture treatment for women with PCOS for both fertility and symptom control.

Main outcome(s): (1) Live birth rate: The patient was followed from confirmation of clinical pregnancy until the pregnancy outcome was achieved. Live birth is defined as delivery of viable infant after 28 weeks of gestation, and the live birth rate is the proportion of women who have a live birth outcome among all pregnant women. (2) Clinical pregnancy rate: Human chorionic gonadotropin (hCG) was positive and the intrauterine pregnancy sac was confirmed by ultrasound. Clinical pregnancy rate is the proportion of women who are identified clinical pregnant during the treatments or follow-up period among all women. (3) Ovulation rate: It was confirmed by ultrasound or increased progesterone. Ovulation rate is the proportion of women who ovulated at least once during the treatments or follow-up period among all women.

INPLASY registration number: This protocol was registered with the International Platform of Registered Systematic Review and Meta-Analysis Protocols (INPLASY) on 12 August 2021 and was last updated on 12 August 2021 (registration number INPLASY202180048).

\section{INTRODUCTION}

Review question / Objective: To assess the effectiveness and safety of CHM combined with acupuncture treatment for women with PCOS for both fertility and symptom control.
Condition being studied: Polycystic Ovary Syndrome (PCOS) is the most common endocrine and metabolic disorder, which affects $5 \%$ to $20 \%$ of women of reproductive age, and it is characterized by irregular menstruation or amenorrhea, ovulatory dysfunction, obesity, 
hyperandrogenism and infertility. At present, the treatment of PCOS is mainly based on oral drugs, which include ovulation induction, insulin sensitizing agents and so on, lifestyle improvement and laparoscopic surgery. It was demonstrated that Chinese herbal medicine (CHM) has a certain effect on improving the symptoms and endocrine metabolism of women with PCOS. In addition, acupuncture may has a important role in ovulation induction via increased $\beta$ endorphin production affecting gonadotropin-releasing hormone (GnRH) secretion in the hypothalamic-pituitaryovarian axis. CHM or acupuncture may be expected to an alternative approach for women with PCOS. There have been some systematic reviews and meta-analyses to evaluate the efficacy of CHM or acupuncture alone in PCOS patients, but there is no evaluation on the efficacy and safety of CHM combined with acupuncture in PCOS patients. We prepare to investigate the effectiveness and safety of CHM combined with acupuncture compared to other therapies for women with PCOS.

\section{METHODS}

Search strategy: We will search publications, with retrieve the date for the self-built database until July 1st, 2021, in nine electronic databases including Wan Fang Data Knowledge Service Platform, China National Knowledge Infrastructure(CNKI), Chinese Scientific Journal Database(VIP database), Chinese Biomedical Literature Database(CBM), PubMed, Web of Science, EMBASE, Cochrane Library and Clinical trial.gov. All publications are without any restriction of countries or languages. We will also hand search the included studies' reference to find out all possible studies. We used the following four groups of search terms as free text and MeSH expressions: 1) 'Medicine, Chinese Traditional' OR 'Drugs, Chinese Herbal' OR 'TCM' OR 'Traditional Chinese Medicine' OR 'Traditional Medicine,Chinese' OR 'Chinese Traditional Medicine' OR 'Chinese Medicine, Traditional' OR 'Chinese Drugs, Plant' OR 'Chinese Herbal Drugs' OR 'Herbal Drugs,
Chinese' OR 'Chinese herbal medicine' OR 'Chinese herbal remedy' OR 'herbal preparations' OR 'herbal remedy' OR 'herbal supplement', OR 'Chinese patent medicine' OR 'traditional Chinese patent medicine' OR 'Chinese patent drugs' OR 'traditional Chinese formula' OR 'traditional Chinese prescription' 2) 'acupuncture' OR 'moxibustion' OR 'Acupuncture Treatment' OR 'Acupuncture Treatments' OR 'Treatment, Acupuncture' OR 'Therapy, Acupuncture' OR 'electroacupuncture' OR 'electro-acupuncture' OR 'electric acupuncture' OR 'Pharmacoacupuncture Treatment' OR 'Treatment, Pharmacoacupuncture' OR 'Pharmacoacupuncture Therapy' OR 'Therapy, Pharmacoacupuncture' OR 'abdominal acupuncture' OR 'ear acupuncture' OR 'needle' OR 'needling' OR 'warming needle moxibustion' OR 'moxabustion' OR 'moxi*' OR 'moxibustion therapy' OR 'acupuncture point' OR 'acupoint' 3) 'Polycystic Ovary Syndrome' OR 'PCOS' OR 'Stein-Leventhal Syndrome' OR 'Stein Leventhal Syndrome' OR 'Syndrome, Stein-Leventhal' OR 'polycystic ovar*' OR 'Ovarian Syndrome, Polycystic' OR 'ovulatory dysfunction' OR 'anovulation' OR 'Amenorrhea' OR 'Amenorrh*' OR 'Oligomenorrhea' OR 'Oligo*' OR 'Infertility' OR 'Sterility' OR 'Reproductive Sterility' OR 'Subfertility' OR 'Subfertile' OR 'SubFertility' OR 'aciesis' OR 'barreness' 4) 'randomized controlled trial' OR 'RCT' OR 'controlled clinical trial' OR 'random*' These terms were translated to Chinese as Chinese search terms. We combined these terms with "And" to finish the search.

Participant or population: Women with PCOS.

Intervention: CHM plus acupuncture.

Comparator: Comparison interventions were no intervention, placebo (including sham acupuncture), lifestyle intervention (e.g. exercise or diet regulation) and conventional medicine treatment of PCOS (e.g. ovulation induction, insulin sensitizing agents,oral contraceptives, estrogenprogestin therapy). 
Study designs to be included: Parallel Randomized Controlled Trials (RCTs).

Eligibility criteria: (1) Types of studiesParallel Randomized Controlled Trials (RCTs); (2) Types of participants Women of reproductive age (18 to 44 years) with PCOS included both women who sought fertility and sought symptom control. We defined PCOS by using the diagnostic criteria of the European Society of Human Reproduction and Embryology (ESHRE) and the American Society of Reproductive Medicine (ASRM) consensus in Rotterdam in 2003. If other diagnostic criteria was used in the original text, the reviewer determine whether the criteria was within the scope of the Rotterdam diagnostic criteria, and we excluded reviews that did not mention specific diagnostic criteria. We excluded individuals who have other aetiologies of hyperandrogenis $m$, such as hyperprolactinemia, androgen-secreting tumour, thyroid disease, Cushing's syndrome, Congenital adrenal hyperplasia. (3) We defined CHM plus acupuncture as the experimental intervention. CHM includes oral preparations such as Chinese herbal medicines, Chinese patent medicines, Chinese medicine granular preparations, hospital preparations. Acupuncture included hand acupuncture, electric acupuncture, warm needling moxibustion, acupuncture plus moxibustion and specific region acupuncture. Fire needling, intradermal needling, three-edge needling, plum blossom needling, laser needling, acupoint injection, pricking blood, transcutaneous electrical nerve stimulation, acupoint embedding are excluded. (4) Comparison interventions were no intervention, placebo (including sham acupuncture), lifestyle intervention (e.g. exercise or diet regulation) and conventional medicine treatment of PCOS (e.g. ovulation induction, insulin sensitizing agents,oral contraceptives, estrogen-progestin therapy).

Information sources: Wan Fang Data Knowledge Service Platform, China National Knowledge Infrastructure(CNKI),
Chinese Scientific Journal Database(VIP database), Chinese Biomedical Literature Database(CBM), PubMed, Web of Science, EMBASE, Cochrane Library and Clinical trial.gov. When the data of publications are insufficient or ambiguous, one of the authors will be in contact with the original author to request detailed about the research by e-mail or telephone or estimating the data.

Main outcome(s): (1) Live birth rate: The patient was followed from confirmation of clinical pregnancy until the pregnancy outcome was achieved. Live birth is defined as delivery of viable infant after 28 weeks of gestation, and the live birth rate is the proportion of women who have a live birth outcome among all pregnant women. (2) Clinical pregnancy rate: Human chorionic gonadotropin (hCG) was positive and the intrauterine pregnancy sac was confirmed by ultrasound. Clinical pregnancy rate is the proportion of women who are identified clinical pregnant during the treatments or follow-up period among all women. (3) Ovulation rate: It was confirmed by ultrasound or increased progesterone. Ovulation rate is the proportion of women who ovulated at least once during the treatments or follow-up period among all women.

Additional outcome(s): (1) Pregnancy loss rate: Pregnancy loss rate is the proportion of women who occur pregnancy loss, which includes early abortion, pregnancy loss in the second or third trimester, biochemical pregnancy and ectopic pregnancy, among all women who conceive. (2) Adverse events: Such as ectopic pregnancy, Ovarian Hyperstimulation Syndrome, follicular luteinization syndrome, and that are defined by the author of the original article. (3) Menstrual cycle: 1) Effective rate: The percentage of women whose menstrual cycle improved among all women. Whether the menstrual cycle improved was determined by the author of the original article. 2) Menstrual cycle (day). (4) Body measurement index (including BMI, waisthip ratio). (5) Sex hormone index (including LH, FSH, LH/FSH, T, E2, SHBG). 
Quality assessment / Risk of bias analysis: Two reviewers will independently assess according to the quality of the research selected by the Cochrane's tool for assessing risk of bias, including the following 7 aspects: random sequence generation (selection bias), allocation concealment (selection bias), blinding of participants and personnel (performance bias), blinding of outcome evaluators (monitoring bias), incomplete result data (friction bias), selective outcome reporting (report bias), and other biases (conflict of interest, sample size), which will be charted and evaluated using the ROB module of Review Manager 5.4

Strategy of data synthesis: RevMan 5.4 software will be used to analyze the included literature. For results with small heterogeneity $\left({ }^{2} 75 \%\right)$, a random-effects model will be used for analysis. Metaanalysis results will be represented by forest maps, while the the number of included studies exceeds 10, a funnel plot is used to assess the potential publication bias.For continuous outcomes, mean difference (MD) will be used with 95\% confidence intervals $(\mathrm{Cl})$. For binary outcomes, the risk ratio (RR) with $95 \%$ confidence intervals $(\mathrm{Cl})$ will be calculated.

Subgroup analysis: Subgroup analysis is performed based on clinical characteristics where data are available.

Sensitivity analysis: If there are enough researches, sensitivity analysis will be performed for the results.

Language: All publications are without any restriction of languages.

Country(ies) involved: China.

Keywords: PCOS; CHM; Acupuncture; meta-analysis.

Contributions of each author:

Author 1 - Xingyan Liang.

Email: xy_liang320@163.com

Author 2 - Yu Su.

Email: suyu7715@163.com
Author 3 - Chunli Lu.

Email: jennylu@bucm.edu.cn

Author 4 - Hongxia Ma.

Email: doctorhongxia@126.com 